\title{
Sorafenib and Mek inhibition is synergistic in medullary thyroid carcinoma in vitro
}

\author{
Yoon Woo Koh ${ }^{1,6}$, Manisha H Shah ${ }^{2}$, Kitty Agarwal ${ }^{3}$, Samantha K McCarty ${ }^{1}$, \\ Bon Seok Koo ${ }^{1,7}$, 'Victoria J Brendel', Chaojie Wang', Kyle Porter ${ }^{4}$, \\ David Jarjoura ${ }^{4}$, Motoyasu Saji' and Matthew D Ringel ${ }^{1}, 2,5$
}

\begin{abstract}
Divisions of ${ }^{1}$ Endocrinology, Diabetes and Metabolism, ${ }^{2}$ Medical Oncology, ${ }^{3}$ Department of Chemistry, ${ }^{4}$ Center for Biostatistics and ${ }^{5}$ Department of Molecular Virology, Immunology and Genetics, The Ohio State University Medical Center, The Ohio State University and The Arthur G. James Cancer Hospital and Richard J. Solove Research Institute, Columbus, Ohio, USA

${ }^{6}$ Department of Otorhinolaryngology, Yonsei University College of Medicine, Seoul, Republic of Korea

${ }^{7}$ Department of Otolaryngology, Head and Neck Surgery, Chungnam National University, Daejoon, Republic of Korea

(Correspondence should be addressed to M D Ringel at 445D McCampbell Hall, 1581 Dodd Drive, Columbus, Ohio 43210, USA; Email: matthew.ringel@osumc.edu)
\end{abstract}

\begin{abstract}
Clinical trials using kinase inhibitors have demonstrated transient partial responses and disease control in patients with progressive medullary thyroid cancer (MTC). The goal of this study was to identify potential combinatorial strategies to improve on these results using sorafenib, a multikinase inhibitor with activity in MTC, as a base compound to explore signaling that might predict synergystic interactions. Two human MTC cell lines, TT and MZ-CRC-1, which harbor endogenous C634W or M918T RET mutations, respectively, were exposed to sorafenib, everolimus, and AZD6244 alone and in combination. 3-(4,5-Dimethylthiazol-2-yl)-2,5-diphenyl tetrasodium bromide (MTT) and poly (ADP-ribose) polymerase (PARP) cleavage assays were performed to measure cell survival and apoptosis. Western blots were performed to confirm activity of the compounds and to determine possible mechanisms of resistance and predictors of synergy. As a solitary agent, sorafenib was the most active compound on MTT assay. Western blots confirmed that sorafenib, everolimus, and AZD6244 inhibited their anticipated targets. At concentrations below its $\mathrm{IC}_{50}$, sorafenib-treated TT and MZ-CRC-1 cells demonstrated transient inhibition and then re-activation of Erk over $6 \mathrm{~h}$. In concordance, synergistic effects were only identified using sorafenib in combination with the Mek inhibitor AZD6244 ( $P<0.001$ for each cell line). Cells treated with everolimus demonstrated activation of Akt and Ret via TORC2 complex-dependent and TORC2 complex-independent mechanisms respectively. Everolimus was neither additive nor syngergistic in combination with sorafenib or AZD6244. In conclusion, sorafenib combined with a Mek inhibitor demonstrated synergy in MTC cells in vitro. Mechanisms of resistance to everolimus in MTC cells likely involved TORC2-dependent and TORC2-independent pathways.
\end{abstract}

Endocrine-Related Cancer (2012) 19 29-38

\section{Introduction}

Medullary thyroid cancer (MTC) arises from parafollicular $\mathrm{C}$ cells, comprises $\sim 5 \%$ thyroid cancers, and presents in hereditary ( $\sim 20 \%$ cases) or sporadic ( $\sim 80 \%$ MTC cases) forms (Kloos et al. 2009). The hereditary form of MTC is associated with multiple endocrine neoplasia type 2 (MEN2), including MEN2A, MEN2B, and familial MTC. Germlineactivating mutations in RET are the cause of inherited forms of MTC and somatic mutations in Ret can be found in 30-50\% of cases of sporadic MTC. For MTC limited to the neck, surgery and in some cases external radiation therapy allow for either cure or disease control in the majority of patients. However, for patients with progressive distant metastases chemotherapy regimens have proven largely ineffective, indicating the need for alternative therapies. One approach that recently has been studied with exciting results is to target the 
constitutively active Ret kinase and/or its key downstream signaling pathways (Wells \& Santoro 2009, Ye et al. 2010).

Mutated Ret in MTC activates several downstream signaling pathways, including the Ras/Raf/Mek/Erk and phosphatidylinositol 3-kinase (PI3K)/Akt/mammalian target of rapamycin (mTOR) cascades resulting in cancer development and perhaps progression (Eng et al. 1996) making it a rational therapeutic target for this disease. Sorafenib is a multikinase inhibitor that blocks activity of Ret kinase, other tyrosine kinases, and Raf serine-threonine kinase members making it a compound of interest in MTC. We recently reported results of a phase 2 clinical trial for patients with advanced MTC in which a partial response rate of $6 \%$ was observed and $50 \%$ of patients demonstrated stable disease $>15$ months, with tumor shrinkage ranging from 8 to 27\% (Lam et al. 2010). However, like other tyrosine kinase inhibitors, most of the patients in this study eventually developed progressive disease (Cabanillas et al. 2010). Thus, we were interested in exploring combinatorial strategies in MTC cells using sorafenib as a base compound due focusing on compounds with logical combinatorial signaling inhibiting characteristics including compounds in clinical trial or already approved for clinical use in the United States. These include the mTOR (TORC1 complex) inhibitor everolimus and the Mek inhibitor AZD6244.

Our results indicate that the antiproliferative activity of sorafenib was synergistically augmented when it was combined with a Mek inhibitor (AZD6244) but not everolimus. This result was predicted by dose-related signaling inhibition experiments using sorafenib alone for both the cell lines. Our data also demonstrate that AZD6244 and everolimus, when used together were not synergistic in either cell line despite inhibition of Mek and TORC1 respectively. Interestingly, everolimus was shown to induce both Ret and Akt phosphorylation and this effect was enhanced by co-treatment with AZD6244, suggesting a possible mechanism of resistance. Taken together, our results underscore the potential of a combined therapeutic approach with sorafenib and Mek inhibitors for the treatment of MTC as well as the need for correlative studies to better define rational combinatorial strategies.

\section{Materials and methods}

\section{Cell lines and reagents}

The human medullary thyroid cancer (MTC) cell lines, TT and MZ-CRC-1, were kindly provided from Bary Nelkin, PhD (Johns Hopkins University, Baltimore,
MD, USA) and Robert Gagel, MD (MD Anderson Cancer Center, Houston, TX, USA) respectively. The TT cells have a heterozygous C634W Ret mutation and the MZ-CRC-1 cells have a heterozygous M918T Ret mutation (personal communications with Drs Nelkin and Gagel and independently confirmed; data not shown). Cells were maintained in RPMI 1640 medium supplemented with heat-inactivated (HI) $20 \%$ fetal bovine serum $(\mathrm{FBS})$ and $1 \times$ nonessential amino acids (1\%; all purchased from Invitrogen Corp.) at $37^{\circ} \mathrm{C}$ and humidified $5 \% \mathrm{CO}_{2}$. For MZ-CRC-1 culture, we used collagen fiber (Collagen I, Rat tail, BD Biosciences, Burlingame, CA, USA) to induce a thin layer on tissue culture surfaces to enhance cell attachment and proliferation. Cells were washed in PBS and placed in RPMI1640 with 2\% FBS (lower serum conditions) in 12-well plates for $24 \mathrm{~h}$ before experiments. All inhibitors were diluted in DMSO as per the manufacturer's recommendations, and control experiments adding equivalent concentrations of DMSO in the absence of inhibitors were performed for each experiment. Sorafenib, everolimus, and tomozolomide for in vitro use were purchased from LC Laboratories (Woburn, MA, USA). AZD6244 for in vitro use was purchased from Selleck Chemicals LLC (Houston, TX, USA).

\section{Protein extraction}

Cells were placed in $10 \mathrm{~cm}$ dishes and cultured until $\sim 50 \%$ confluent. After washing with PBS, cells were cultured in fresh medium with $2 \%$ FBS for $24 \mathrm{~h}$, and experiments were performed with blockers at the concentrations and time points noted. To stop the experiments, cells were rinsed twice with $10 \mathrm{ml}$ of ice-cold PBS, scraped, transferred to $1.5 \mathrm{ml}$ tubes, and centrifuged $(500 \mathrm{~g})$. Cells were lysed in M-PER buffer (Invitrogen) with $1 \mu \mathrm{g} / \mathrm{ml}$ aprotonin, $1 \mu \mathrm{g} / \mathrm{ml}$ leupeptin, $1 \mu \mathrm{g} / \mathrm{ml}$ pepstatin $\mathrm{A}, 20 \mu \mathrm{M}$ 4-amidino-phenyl methane-sulfonyl fluoride (APMSF) and $0.3 \mathrm{mM}$ okadaic acid (EMD, Gibbstown, NJ, USA). The tubes were centrifuged at $12000 \mathrm{~g}$ for $10 \mathrm{~min}$ at $4{ }^{\circ} \mathrm{C}$. The supernatant was saved and stored at $-80^{\circ} \mathrm{C}$. Protein content of the supernatant was quantified using a BSA Kit (Pierce Biotechnology, Inc., Rockford, IL, USA).

\section{Immunoblotting and protein detection}

Primary antibodies used in the studies include those directed against: phospho-Ret (tyrosine 905), total Akt, phospho-Akt (Ser 473), Erk, phospho-Erk, p70S6K, phospho-p70S6K, Rictor (53A2) (Cell Signaling Technology, Beverly, MA, USA), PARP (F-2), and total Ret (c-19) (Santa Cruz Biotechnology, Inc., Santa Cruz, CA, USA). Data were normalized relative to 
protein levels of GAPDH (Novus Biologicals, Inc., Littleton, CO, USA), which was probed by polyclonal rabbit antibody (1:1000, Cell Signaling Technology). For western blots, $20 \mu \mathrm{g}$ of total protein lysate was suspended in reduced SDS sample buffer and boiled for $5 \mathrm{~min}$. Protein lysates were subjected to SDS-PAGE $(8 \%)$, and the separated proteins were transferred to nitrocellulose membranes $(0.45 \mu \mathrm{m}$ pore size, Invitrogen) by electrophoretic blotting (Invitrogen). Nonspecific binding was prevented by blocking with $0.1 \%$ Tween 20 in PBS (PBS-T) containing 5\% nonfat dry milk overnight at $4{ }^{\circ} \mathrm{C}$. Immunoblotting was performed in the following manner: membranes were washed four times (10 min/wash) with PBS-T, incubated with the primary antibody in PBS-T containing $5 \%$ BSA or nonfat dry milk for overnight at $4{ }^{\circ} \mathrm{C}$, and washed four times with PBS-T (15 min/wash). Membranes were then incubated with the secondary antibody conjugated with peroxidase (Santa Cruz Biotechnologies) in PBS-T containing 5\% nonfat dry milk for $1 \mathrm{~h}$ at room temperature. After washing with PBS-T four times (15 min/wash), protein bands on the blots were visualized using ECL Plus Western Blotting Detection Reagents (GE Healthcare Bio-Science Corpoation, Piscataway, NJ, USA). All western blot experiments were repeated in independent experiments to confirm results.

\section{Cell growth studies}

Cell survival and proliferation was determined by 3-(4,5-dimethylthiazol-2-yl)-2,5-diphenyl tetrasodium bromide (MTT; Sigma) assay. Cells were plated in 96-well plates and grown until 50\% confluence was reached, after which medium was replaced daily in all experiments. Each experiment was performed three times in triplicate. Ten microliters of $5 \mathrm{mg} / \mathrm{ml}$ MTT assay was added to each well, and the cells were subsequently returned to the incubator for $4 \mathrm{~h}$. Isopropanol with $0.04 \mathrm{~N} \mathrm{HCl}(100 \mu \mathrm{l})$ was added, and absorbance on a 96-well plate with a wavelength of $570 \mathrm{~nm}$ was measured (Revelation 4.02, DYNEX Technology, Chantilly, VA, USA). To generate doseresponse curves for each cell line, MTT absorbance was determined 3 days after exposure to either single agent or combination therapy. For growth analyses, cells were treated daily with indicated doses suspended in fresh media.

\section{siRNA studies}

Specific siRNA for Rictor and scrambled siRNA control were obtained from Thermo Scientific Dharmacon Products (Lafayette, CO, USA). When
MZ-CRC-1 cells reached $80 \%$ confluent, the medium was aspirated and cells were washed twice with PBS. Cells were then incubated with $1.2 \mathrm{nmol}$ of siRNA and Lipofectamine 2000 (Invitrogen) in OptiMEM medium (Invitrogen) for $16 \mathrm{~h}$ in a humidified $5 \% \mathrm{CO}_{2}$ incubator overnight. After incubation, the OptiMEM medium was aspirated and the RPMI medium containing 2\% HI FBS was added to culture dishes. After $24 \mathrm{~h}$, the medium was switched to fresh medium for $3 \mathrm{~h}$ and $1 \mu \mathrm{M}$ everolimus or DMSO was added for control. After $1 \mathrm{~h}$ of incubation, proteins were isolated from cells as described above and western blots were performed.

\section{Statistical analysis}

Measurements of DNA content and MTT assays were repeated at least three times in triplicate. Values are the mean \pm s.D. of these experiments. All western blot experiments were repeated on at least three separate occasions to confirm results. The presence of synergy was assessed in the following manner: Mixed-effect linear models were fit to the MTT optical densities. The models contained main effects for each individual drug concentration (three per drug per experiment) and interaction effects for each combination of concentrations (nine per experiment). Random plate effects were included to account for potential dependencies among observations from the same plate. Each hypothesis was tested as a single contrast of model coefficients. The synergy hypothesis for each was that the combination effect (e.g. control-sorafenib/ AZD6244) would not be greater than the sum of effects from the individual agents (e.g. (controlsorafenib $)+($ control-AZD6244)). All dose levels were below the $\mathrm{IC}_{50}$ to avoid a ceiling effect and increase the power to test this synergy hypothesis. Each a priori hypothesis was unidirectional; therefore each combination was evaluated by a one-sided singlecontrast hypothesis test. Bonferroni adjustments were used to control for multiple testing, resulting in each hypothesis being evaluated at $\alpha=0.008$.

Table 1 Cell viability $\mathrm{IC}_{50}$ values for individual compounds in both MTC cell lines based on MTT assays

\begin{tabular}{lcc}
\hline Compound & TT cells $(\mu \mathrm{M})$ & MZ-CRC-1 cells $(\mu \mathrm{M})$ \\
\hline Sorafenib & $0.17 \pm 0.04$ & $6.87 \pm 0.13$ \\
Everolimus & $6.39 \pm 0.75$ & $14.69 \pm 0.28$ \\
AZD6244 & $6.35 \pm 1.07$ & $>40$ \\
\hline
\end{tabular}

Values were obtained for experiments performed in duplicate on more than three separate occasions. 


\section{Results}

\section{Sorafenib inhibits cell growth at lower concen- trations than everolimus, and AZD6244; TT cells are more sensitive than MZ-CRC-1 cells}

To measure the growth-inhibitory action of sorafenib, everolimus, temozolomide, and AZD6244 in MTC cells in vitro, we performed MTT assays, using single agent alone for 3 days. For each cell line, the $\mathrm{IC}_{50}$ for cell viability was determined in experiments using a 3-day continuous exposure to single agent. The cell viability $\mathrm{IC}_{50}$ of sorafenib in $\mathrm{TT}$ vs MZ-CRC-1 cells differed by $\sim 40$-fold ( 0.17 vs $6.87 \mu \mathrm{M}$ respectively), although this was the most active compound for both the cell lines (Table 1). Similarly, the cell viability $\mathrm{IC}_{50}$ of everolimus was twofold higher in MZ-CRC-1 than in TT cells (10 vs $5 \mu \mathrm{M}$ respectively; Table 1 ). The cell viability $\mathrm{IC}_{50}$ of AZD6244 for TT cells was $5 \mu \mathrm{M}$ (Table 1); however, an $\mathrm{IC}_{50}$ was never achieved with this agent in MZ-CRC-1 cells, even with concentrations as high as $40 \mu \mathrm{M}$ (Fig. 1A). Inhibition of cell growth, following temozolomide treatment was not achieved for either cell line (data not shown).
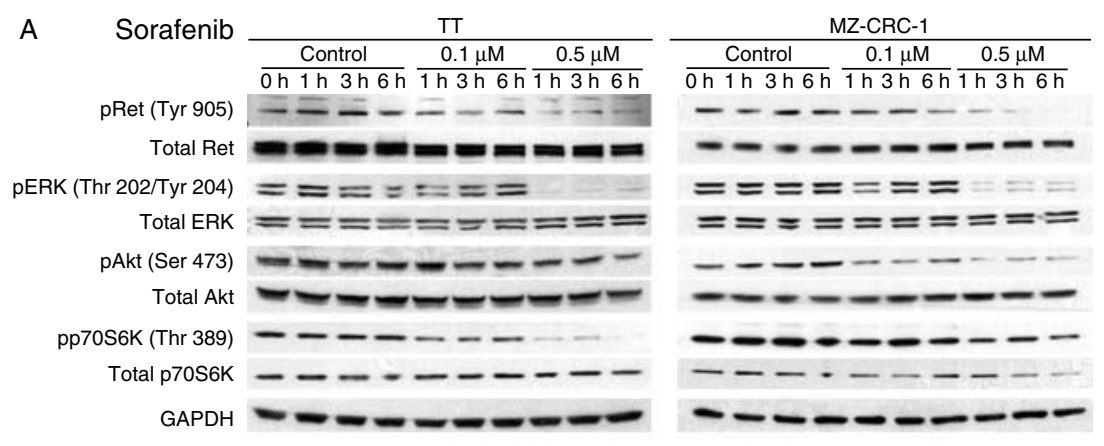

B

Everolimus $\frac{\mathrm{TT}}{\frac{\text { Control }}{0 \mathrm{~h} \mathrm{1h3h6h}} \frac{1 \mu \mathrm{M}}{1 \mathrm{~h} \mathrm{3h6h} \frac{5 \mu \mathrm{M}}{1 \mathrm{~h} \mathrm{3h} 6 \mathrm{~h}}}}$
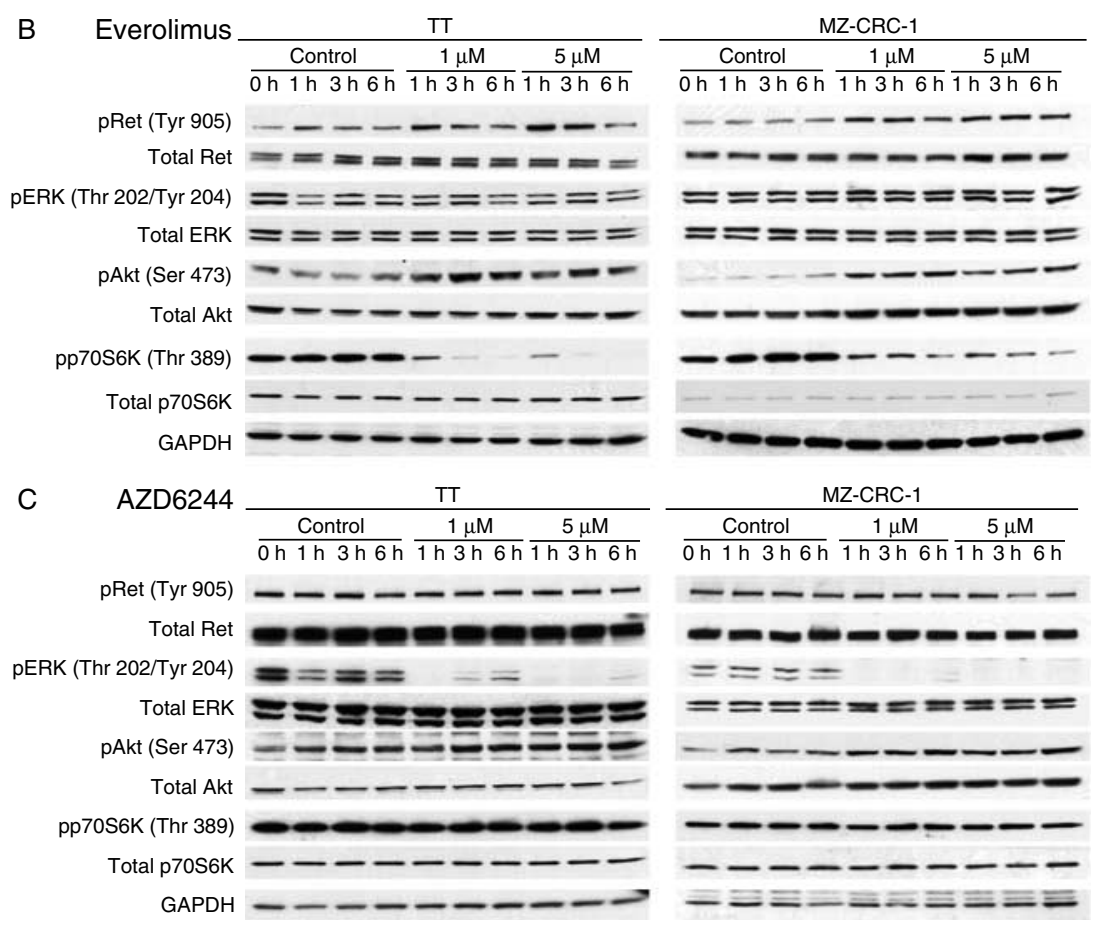

Figure 1 Individual agent-signaling inhibition. (Panel A) TT cells were treated with 0.1 and $0.5 \mu \mathrm{M}$ of sorafenib for 1,3 , and $6 \mathrm{~h}$. Sorafenib inhibits phosphorylation of Ret, Erk, and p70S6 kinase. Akt inhibition was incomplete in the TT cell line and did not correlate with loss of cell number. (Panel B) MZ CRC1 cells were treated with sorafenib at the same doses and times as for TT cells. In both the cell lines, $0.1 \mu \mathrm{M}$ sorafenib inhibits $\mathrm{pErk}$ at $1 \mathrm{~h}$ and then the inhibition is lost, while the other pathways remain suppressed.

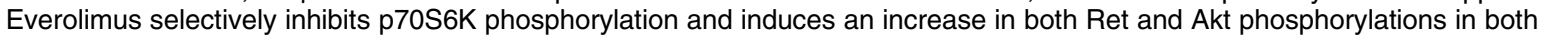
the cell lines. (Panel C) AZD6244 selectively inhibits Erk phosphorylation. There is a modest induction of Akt phosphorylation that is noted. 


\section{Pathway inhibition of inidividual Ret, Mek, and mTOR inhibitors in MTC cells}

Sorafenib reduced levels of phospho-Ret, phosphoErk, phospho-Akt, and phospho-p70S6 kinase in both TT and MZ-CRC-1 cells as would be predicted based on the known targets of the compound (Fig. 1A). Interestingly, the level of phospho-Erk was reduced beginning at concentrations of $0.1 \mu \mathrm{M}$ in both the cell lines as early as $1 \mathrm{~h}$ after treating the cells, but phosphorylated Erk was detectable after $3 \mathrm{~h}$ of treatment and levels returned to pre-exposure levels after $6 \mathrm{~h}$ despite continuous exposure to the compound. Erk activation was completely inhibited at $0.5 \mu \mathrm{M}$ dosing in both the cell lines. The total Erk expression remained the same during all the treatments. This escape from sorafenib signaling inhibition was not seen consistently for phosphorylated Akt, phosphorylated p70S6 kinase (phospho-p70S6K; Fig. 1A), or p38 Map kinase (data not shown). As predicted, western blots after everolimus treatment show only a significant decrease in phospho-p70S6K, a direct downstream target of mTOR (Fig. 1B), and AZD6244 induced a significant decrease in phospho-Erk beginning at concentrations of $1 \mu \mathrm{M}$ without inhibiting other pathways (Fig. 1C). While both the compounds elicited an increase in levels of serine 473 phosphorylated Akt, everolimus also induced Ret phosphorylation. Taken together, the data suggest that at doses below the cell viability $\mathrm{IC}_{50}$, sorafenib only transiently inhibited Erk phosphorylation, suggesting that maintenance of this inhibition may be beneficial in enhancing the biological effects of this compound. They also suggest that the relative resistance to everolimus and AZD6244 as solitary agents may involve activation of Ret or Akt.

\section{Sorafenib is synergistic with AZD6244 in both the cell lines; other combinations were non-synergistic}

To determine, whether the western blot analysis of sorafenib therapy predicted synergy, combination
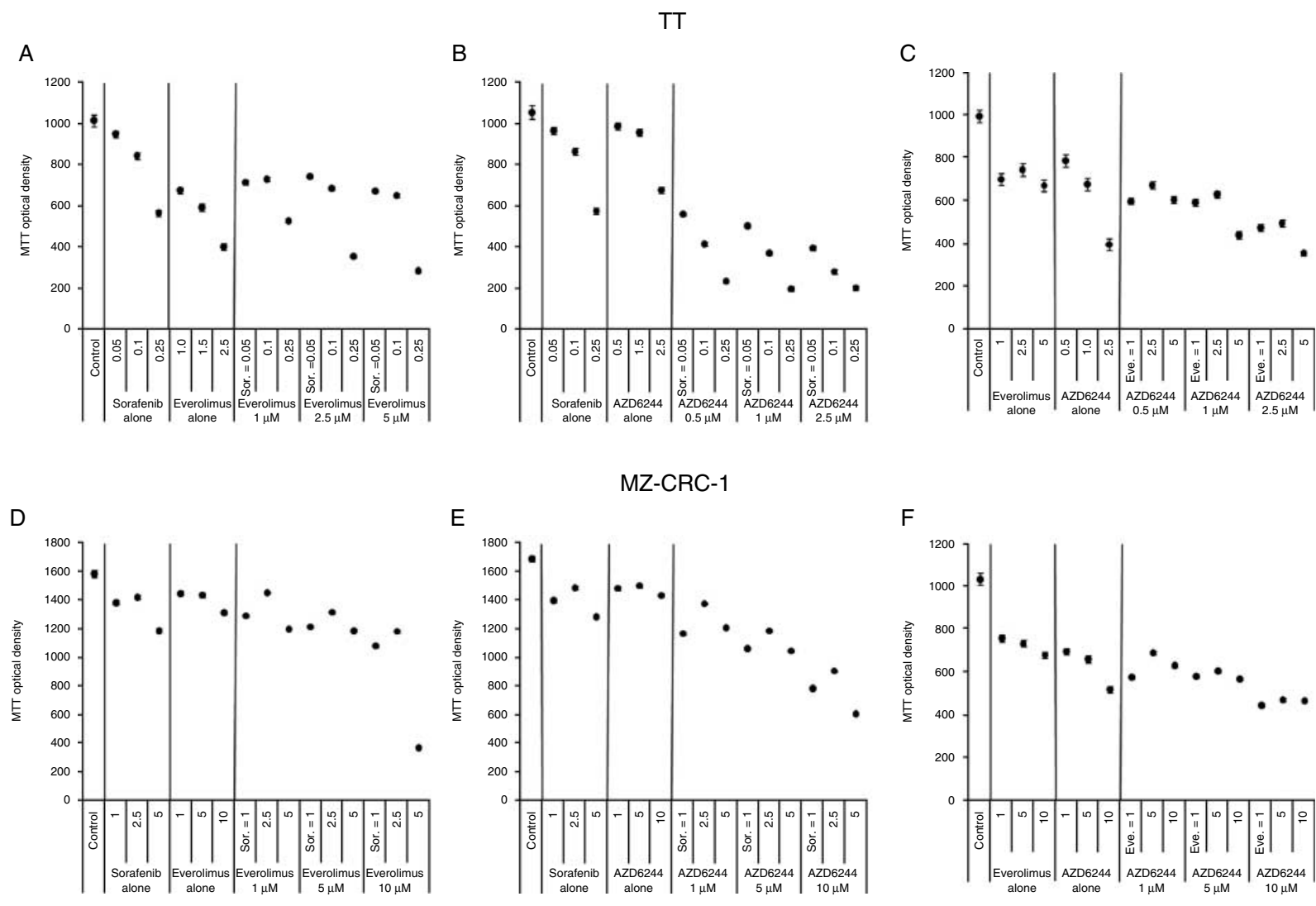

Figure 2 Combination therapy in MTC cells. TT (Panels A-C) and MZ-CRC-1 (Panels D-F) cells were treated with each compound alone and in combination with sorafenib (Sor), AZD 6344, and/or everolimus. All potential two-compound combinations were examined and experiments were performed in which each individual compound was kept at a constant concentration or varied. Synergy in both the cell lines was detected only with the combination of sorafenib and AZD6244 (Panels B and E, $P<0.001$ for both) with the effect being more pronounced for the TT cell line. Data points are estimated means from the mixed-effect model and the error bars are standard error based on residual variance from the mixed-effect model. 
studies were performed using concentrations of sorafenib below and at the cell viability $\mathrm{IC}_{50}$ (Table 1) for both the cell lines. In these studies, combination of low-dose sorafenib along with doses of AZD6244 below its individual $\mathrm{IC}_{50}$ induced significantly greater inhibition of TT and MZ-CRC-1 cell growth compared with either agent alone that was synergistic on statistical analysis $(P<0.001$ for both the cell lines, Fig. 2B and E). The synergistic effect was less pronounced in the MZ-CRC-1 cell line and only became cytotoxic (reduction in cell number below baseline) at higher concentrations. By contrast, the combination of sorafenib and everolimus did not elicit significantly greater inhibition of TT and MZ-CRC-1 cell growth compared with either agent alone (Fig. 2A and D). Also, everolimus and AZD6244 combination treatment was not synergistic (Fig. $2 \mathrm{C}$ and F). These data suggest that loss of Erk inhibition may be responsible in part for the loss of sorafenib effect at low doses and that this can be exploited with therapeutic intent for combination therapies.

\section{Combination therapy signaling}

Next, we wanted to confirm that the combination therapies were inhibiting the anticipated targets by western blot. Combination therapy with sorafenib and AZD6244 for $3 \mathrm{~h}$ resulted in inhibition of Ret and Erk activites at low concentations that was maintained for both the cell lines, consistent with the synergistic results in the MTT assay (Fig. 3). Everolimus and AZD6244 alone and in combination effectively inhibited their respective target pathways in both the cell lines (Figs 1B, C and 3); however, everolimus and
AZD6244 treatment caused increased phosphorylation of Akt Ser473 in both the cell lines (Figs 1 and 3). These results are consistent with feedback activation of Akt in response to mTOR, or Mek inhibition as full activity of Akt requires phosphorylation at Ser473 by mTORC2 (Carracedo et al. 2008, Kinkade et al. 2008, Jin et al. 2009, Huynh et al. 2010). Surprisingly, everolimus treatment also induced an increase in phosphorylated Ret in both the cell lines (Figs 1C and 3). Notably, in combination, these agents resulted in a more striking activation of p-Ret, as well as activation of p-Akt cells (Figs 1 and 3). Triple combination therapy abolished this effect. Taken along with the MTT results, the data suggest that persistent inhibition of both Ret and Erk may be needed for synergistic effects in the TT and MZ-CRC-1 cell lines.

\section{mTOR inhibitor-induced Akt activation can be partially abrogated by inhibition of Rictor; Ret phosphorylation is unaffected}

To determine, whether activation of the TORC2 complex was involved in everolimus-induced Akt and Ret phosphorylation, we reduced Rictor expression using siRNA. In MZ-CRC-1 cells, reduced levels of Rictor achieved by siRNA transfection decreased everolimus-induced Akt activation vs cells transfected with control scrambled siRNA. By contrast, the level of induced phospho-Ret was not altered by the Rictor siRNA (Fig. 4). These data suggest that TORC2independent mechanisms are involved in secondary phosphorylation of Ret in the MTC cells.

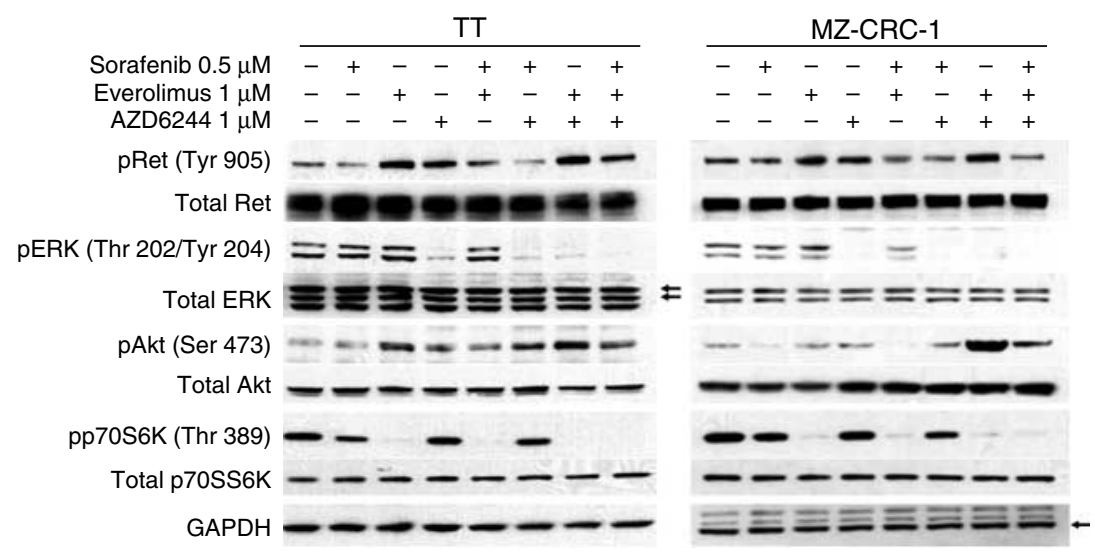

Figure 3 Cell signaling inhibition of combination therapy. Western blots were performed at the listed concentrations for each cell line after $3 \mathrm{~h}$ of exposure to confirm that the combinations of therapies were active in vitro. Each combination inhibited the expected targets; combined inhibition of Ret and Erk phosphorylation required the combination of AZD6244 and sorafenib in both the cell lines. Everolimus-induced Ret and Akt phosphorylation was enhanced by co-treatment with AZD6244. The arrows in the ERK blot point to the dual ERK bands. 


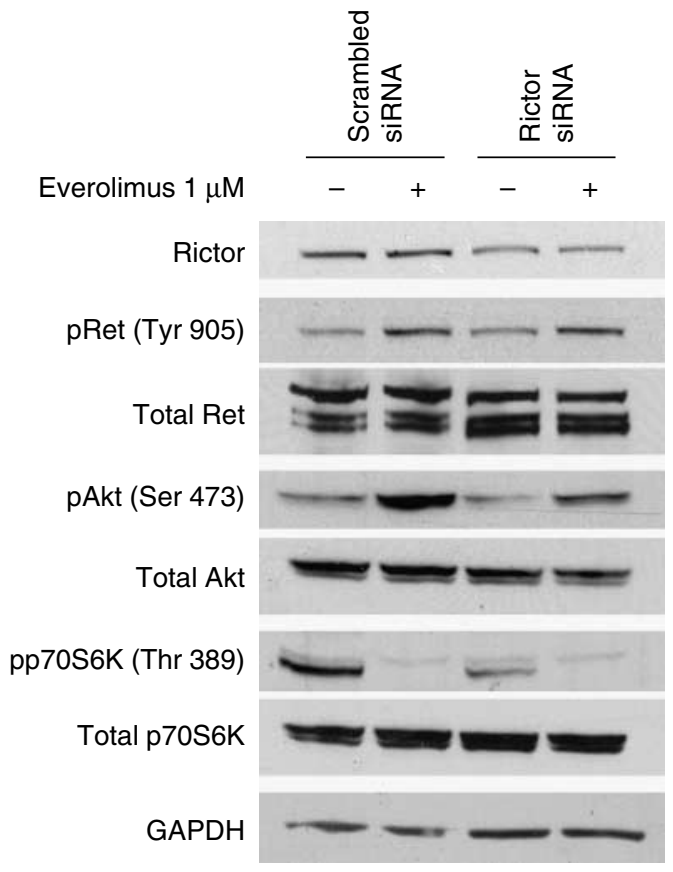

Figure 4 Everolimus-induced Akt activation, but not Ret activation, is dependent on the mTORC2 complex. MZ-CRC-1 cells were transfected with control (scrambled) and Rictor siRNAs. Rictor SiRNA reduced Rictor levels by $\sim 50 \%$.

Everolimus-induced Akt phosphorylation was partially inhibited while the level of phospho-Ret was unaffected by Rictor siRNA.

\section{Discussion}

The development of effective treatments with metastatic progressive MTC is needed for these patients as they have an $\sim 50 \%$ 5-year mortality rate (Kloos et al. 2009). Sorafenib and other kinase inhibitors that target Ret along with other kinases have proven to have substantial albeit transient clinical activity in these patients, underscoring the importance of this signaling pathway in tumor progression (Sherman 2009, Wells \& Santoro 2009). Because of the transient and incomplete nature of the reported responses, a better understanding of feedback mechanisms and ultimately the development of combinatorial treatment strategies likely will be needed to improve treatments further.

This study was performed to identify potential pathways of escape from sorafenib at subtherapeutic concentrations and to determine if these data predicted synergistic or additive combinatorial activity. We focused on several pathways for which agents are in clinical trial for thyroid cancer and have been previously analyzed in preclinical studies. For example, sorafenib in combination with an mTOR or Mek inhibitor, has been reported to have potent antitumor activity in other cancers including hepatocellular and gastric cancers (Yang et al. 2009, Huynh et al. 2010). In addition, simultaneous inhibition of the PI-3K/Akt/mTOR and ras/raf/Mek/ Erk signaling pathways is effective in vitro and in animal models (Bedogni et al. 2004, Legrier et al. 2007, Kinkade et al. 2008, Jin et al. 2009, Huynh et al. 2010). However, to our knowledge the combinations analyzed herein have not been reported previously in MTC.

We found that the cell viability $\mathrm{IC}_{50}$ for sorafenib in the MZ-CRC-1 cells with a Ret M918T point mutation was higher than the $\mathrm{IC}_{50}$ for TT cells with a Ret $\mathrm{C} 634 \mathrm{~W}$ point mutation. The inhibitory effect of sorafenib we observed was not predominantly apoptotic based on western blots for PARP cleavage for both cell lines and also using FACS for MZ-CRC-1 cells (data not shown). These results are consistent with those obtained for Ret kinase inhibition by sorafenib using models in which fibroblasts were transfected with Ret 634 and 918 mutants (Carlomagno et al. 2006). However, it is notable that the inhibition of Ret, Erk, and Akt phosphorylation by sorafenib was similar between the two cell lines despite the differences in the effects on cell viability suggesting that the mechanisms behind the difference in sensitivity in the two cell lines may relate to other differences between the cells or the Ret mutants.

It is of interest that everolimus treatment resulted in enhanced phosphorylation of Ret in both the cell lines. Everolimus inhibits only the TORC1 complex that is responsible for phosphorylating p70S6K and other targets. It is well recognized that TORC1 inhibitors can cause a secondary increase in serine 473 phosphorylation of Akt due to feedback by the TORC 2 complex responsible for Akt phosphorylation at that site in some cell systems (Carracedo et al. 2008). This appears to be the case in the MTC cells. Indeed, selective disruption of the TORC2 complex using a Rictor siRNA reduced Akt serine 473 phosphorylation. However, the Rictor siRNA had no effect on everolimus-induced Ret phosphorylation, suggesting alternative feedback loops for this receptor. Upregulation of receptor tyrosine kinase such as platelet-derived growth factor receptors (PDGFRs) and insulin-like growth factor 1 receptors have been reported following mTOR inhibition through incompletely defined mechanisms (Zhang et al. 2007, Carracedo et al. 2008). However, in our case, the Ret proteins are constitutively activated, suggesting that further activation can occur through mTOR inhibition. No change in Ret protein levels was identified on western blot. Further studies are needed to better clarify this mechanism. Contrary to previous reports in other cell systems (Carracedo 
et al. 2008, Jin et al. 2009), everolimus treatment did not induce the MAPK activation in these cells, as measured by Thr202/Tyr204 pErk levels.

In this study, the cell viability $\mathrm{IC}_{50}$ of sorafenib for TT cells carrying Ret C634 point mutation was $0.17 \mu \mathrm{M}$ and inhibition of Erk was lost at lower concentrations. Synergy was achieved by combining sorafenib with a Mek inhibitor that allowed for maintenance of Erk inhibition. These data emphasize the importance of this signaling cascade in survival of these MTC cells. However, because AZD6244 alone was ineffective, and the combination was cytostatic until higher concentrations were used, it is likely that other pathways are also important in the antiproliferative effect of sorafenib in vitro. Additional pathways known to be inhibited by sorafenib that may be active in vivo include vascular endothelial growth factor receptors and PDGFRs. These were not studied in this in vitro study. Similar observations have been shown in response to Mek inhibitors in other cell systems. For example, Yoon et al. (2009) reported that Akt was activated through the EGFR/HER3 /PI3K pathway following AZD6244 treatment in gastric cancer cells. Therefore, we suspected that Akt activation during Mek inhibition might be associated with resistance to Mek inhibitor in a mTORindependent manner, since there was no synergy between everolimus and AZD6244 in the MTC cells. Indeed, combination treatment with Mek and PI3K inhibitors has been reported previously to be beneficial in other tumor types. This synergy likely involves pathways other than mTOR, since the combination of everolimus and AZD6244 was not synergistic in our experiments.

Because western blot analysis showed that the levels of phospho-Erk returned to pre-exposure levels after the cells had been treated for $6 \mathrm{~h}$ at concentrations of $0.1 \mu \mathrm{M}$ sorafenib in both the cell lines, we hypothesized that inhibition of Erk signaling pathway by AZD6244 would enhance the antitumor activity of sorafenib. Indeed, the combination of sorafenib and Mek inhibitor AZD6244 was synergistic in both the cell lines. Based on these data, sorafenib and Mek inhibitors together may have promise in treating MTC patients particularly with Ret C634 point mutation. While this study was limited to in vitro observations, Yang et al. (2009) observed that treatment of gastric cancer xenografts with sorafenib triggers phosphorylation of Erk. They further showed that such combination leads to inhibition of tumor cell proliferation and increased apoptosis. The combination of sorafenib and AZD6244 was also shown to be effective in vivo in hepatocellular carcinoma models (Huynh et al. 2010).
Recent data suggest that inhibition of Raf kinases may, in the setting of an activated wild-type Braf protein, lead to enhanced signaling through Raf isoform heterodimers and subsequent activation of Erk (Poulikakos et al. 2010). It is also possible that loss of expression or function of the dual specificity MAPK phosphatases could also be involved in the restoration of Erk activity following sorafenib therapy. In addition, the role of specific downstream effectors of Erk in resistance or sensitivity to its inhibition in MTC cells requires further exploration. The data, however, provide a rationale for further exploring combined Ret, Raf, Erk inhibiting compounds in MTC therapy in vivo. Indeed, the combination of sorafenib and AZD6244 is currently being studied in a phase I/II clinical trial in advanced hepatocellular carcinoma (www.clinicaltrials.gov).

To our knowledge, this study is the first to demonstrate that mTORC1 inhibition can enhance phosphorylation of constitutively activated Ret. Our findings have important implications for MTC therapy. It was predicted that tumors with hyperactive mTORC1 would be sensitive to mTOR inhibition. However, the discovery of an mTORC1-PI3K feedback loop (Harrington et al. 2004, 2005, Shah et al. 2004, O'Reilly et al. 2006), and now the identification of what is to our knowledge a previously undescribed negative feedback loop regulating Ret, raises the question of whether this feedback may be detrimental to the efficacy of rapamycin and its analogs in MTC monotherapy or could be exploited in further combination therapy studies.

In summary, our data suggest that the combination of a Mek inhibitor AZD6244 with sorafenib may represent a promising strategy to further explore in vivo. The data also point to new mechanisms of therapeutic resistance through feedback-enhanced activation of constitutively active Ret kinases that may need to be considered in future strategies.

\section{Declaration of interest}

Dr Ringel has previously served on an advisory board for Veracyte and has been on an advisory panel for AstraZeneca; neither of which were involved in the experiments presented in this manuscript nor in funding the work.

\section{Funding}

This work was supported by a grant from NIH (CA P0124570) to M D Ringel. 


\section{References}

Bedogni B, O'Neill MS, Welford SM, Bouley DM, Giaccia AJ, Denko NC \& Powell MB 2004 Topical treatment with inhibitors of the phosphatidylinositol $3^{\prime}$-kinase/Akt and Raf/mitogen-activated protein kinase kinase/extracellular signal-regulated kinase pathways reduces melanoma development in severe combined immunodeficient mice. Cancer Research 64 2552-2560. (doi:10.1158/0008-5472.CAN-03-3327)

Cabanillas ME, Waguespack SG, Bronstein Y, Williams MD, Feng L, Hernandez M, Lopez A, Sherman SI \& Busaidy NL 2010 Treatment with tyrosine kinase inhibitors for patients with differentiated thyroid cancer: the M. D. Anderson experience. Journal of Clinical Endocrinology and Metabolism 95 2588-2595. (doi:10. 1210/jc.2009-1923)

Carlomagno F, Anaganti S, Guida T, Salvatore G, Troncone G, Wilhelm SM \& Santoro M 2006 BAY43-9006 inhibition of oncogenic RET mutants. Journal of National Cancer Institute 98 326-334. (doi:10.1093/jnci/djj069)

Carracedo A, Ma L, Teruya-Feldstein J, Rojo F, Salmena L, Alimonti A, Egia A, Sasaki AT, Thomas G, Kozma SC et al. 2008 Inhibition of mTORC1 leads to MAPK pathway activation through a PI3K-dependent feedback loop in human cancer. Journal of Clinical Investigation 118 3065-3074. (doi:10.1172/JCI34739)

Eng C, Clayton D, Schuffenecker I, Lenoir G, Cote G, Gagel RF, van Amstel HK, Lips CJ, Nishisho I, Takai SI et al. 1996 The relationship between specific RET protooncogene mutations and disease phenotype in multiple endocrine neoplasia type 2. International RET mutation consortium analysis. Journal of the American Medical Association 276 1575-1579. (doi:10.1001/jama.1996. 03540190047028)

Harrington LS, Findlay GM, Gray A, Tolkacheva T, Wigfield S, Rebholz H, Barnett J, Leslie NR, Cheng S, Shepherd PR et al. 2004 The TSC1-2 tumor suppressor controls insulin-PI3K signaling via regulation of IRS proteins. Journal of Cell Biology 166 213-223. (doi:10. 1083/jcb.200403069)

Harrington LS, Findlay GM \& Lamb RF 2005 Restraining PI3K: mTOR signalling goes back to the membrane. Trends in Biochemical Sciences 30 35-42. (doi:10.1016/j. tibs.2004.11.003)

Huynh H, Ngo VC, Koong HN, Poon D, Choo SP, Toh HC, Thng CH, Chow P, Ong HS, Chung A et al. 2010 AZD6244 enhances the anti-tumor activity of sorafenib in ectopic and orthotopic models of human hepatocellular carcinoma (HCC). Journal of Hepatology 52 79-87. (doi:10.1016/j.jhep.2009.10.008)

Jin N, Jiang T, Rosen DM, Nelkin BD \& Ball DW 2009 Dual inhibition of mitogen-activated protein kinase kinase and mammalian target of rapamycin in differentiated and anaplastic thyroid cancer. Journal of Clinical Endocrinology and Metabolism 94 4107-4112. (doi:10.1210/ jc.2009-0662)
Kinkade CW, Castillo-Martin M, Puzio-Kuter A, Yan J, Foster TH, Gao H, Sun Y, Ouyang X, Gerald WL, Cordon-Cardo C et al. 2008 Targeting AKT/mTOR and ERK MAPK signaling inhibits hormone-refractory prostate cancer in a preclinical mouse model. Journal of Clinical Investigation 118 3051-3064. (doi:10.1172/ JCI34764)

Kloos RT, Eng C, Evans DB, Francis GL, Gagel RF, Gharib H, Moley JF, Pacini F, Ringel MD, Schlumberger M et al. 2009 Medullary thyroid cancer: management guidelines of the American Thyroid Association. Thyroid 19 565-612. (doi:10.1089/thy.2008.0403)

Lam ET, Ringel MD, Kloos RT, Prior TW, Knopp MV, Liang J, Sammet S, Hall NC, Wakely PE Jr, Vasko VV et al. 2010 Phase II clinical trial of sorafenib in metastatic medullary thyroid cancer. Journal of Clinical Oncology 28 2323-2330. (doi:10.1200/JCO.2009.25.0068)

Legrier ME, Yang CP, Yan HG, Lopez-Barcons L, Keller SM, Perez-Soler R, Horwitz SB \& McDaid HM 2007 Targeting protein translation in human non small cell lung cancer via combined MEK and mammalian target of rapamycin suppression. Cancer Research $\mathbf{6 7}$ 11300-11308. (doi:10.1158/0008-5472.CAN-07-0702)

O'Reilly KE, Rojo F, She QB, Solit D, Mills GB, Smith D, Lane H, Hofmann F, Hicklin DJ, Ludwig DL et al. 2006 mTOR inhibition induces upstream receptor tyrosine kinase signaling and activates Akt. Cancer Research 66 1500-1508. (doi:10.1158/0008-5472.CAN05-2925)

Poulikakos PI, Zhang C, Bollag G, Shokat KM \& Rosen N 2010 RAF inhibitors transactivate RAF dimers and ERK signalling in cells with wild-type BRAF. Nature $\mathbf{4 6 4}$ 427-430. (doi:10.1038/nature08902)

Shah OJ, Wang Z \& Hunter T 2004 Inappropriate activation of the TSC/Rheb/mTOR/S6K cassette induces IRS1/2 depletion, insulin resistance, and cell survival deficiencies. Current Biology 14 1650-1656. (doi:10.1016/j. cub.2004.08.026)

Sherman SI 2009 Advances in chemotherapy of differentiated epithelial and medullary thyroid cancers. Journal of Clinical Endocrinology and Metabolism 94 1493-1499. (doi:10.1210/jc.2008-0923)

Wells SA Jr \& Santoro M 2009 Targeting the RET pathway in thyroid cancer. Clinical Cancer Research 15 7119-7123. (doi:10.1158/1078-0432.CCR-08-2742)

Yang S, Ngo VC, Lew GB, Chong LW, Lee SS, Ong WJ, Lam WL, Thng CH, Koong HN, Ong HS et al. 2009 AZD6244 (ARRY-142886) enhances the therapeutic efficacy of sorafenib in mouse models of gastric cancer. Molecular Cancer Therapeutics 8 2537-2545. (doi:10. 1158/1535-7163.MCT-09-0213)

Ye L, Santarpia L \& Gagel RF 2010 The evolving field of tyrosine kinase inhibitors in the treatment of endocrine tumors. Endocrine Reviews 31 578-599. (doi:10.1210/er. 2009-0031)

Yoon YK, Kim HP, Han SW, Hur HS, Oh DY, Im SA, Bang YJ \& Kim TY 2009 Combination of EGFR and MEK1/2 
inhibitor shows synergistic effects by suppressing EGFR/HER3-dependent AKT activation in human gastric cancer cells. Molecular Cancer Therapeutics 8 2526-2536. (doi:10.1158/1535-7163.MCT09-0300)

Zhang L, Huang J, Yang N, Greshock J, Liang S, Hasegawa K, Giannakakis A, Poulos N, O'BrienJenkins A, Katsaros D et al. 2007 Integrative genomic analysis of phosphatidylinositol $3^{\prime}$-kinase family identifies PIK3R3 as a potential therapeutic target in epithelial ovarian cancer. Clinical Cancer Research 13 5314-5321. (doi:10.1158/1078-0432.CCR-06-2660)

Received in final form 12 November 2011 Accepted 22 November 2011 Made available online as an Accepted Preprint 22 November 2011 